\title{
Branch retinal artery occlusion following carotid stenting: A case report
}

\author{
RAN SUN ${ }^{1,2}$, XIAO-YAN PENG ${ }^{2}$, QI-SHENG YOU ${ }^{2}$, LI-QUN JIAO $^{3}$ and DA-CHUAN LIU ${ }^{1}$ \\ ${ }^{1}$ Department of Opthalmology, Beijing Xuan Wu Hospital, Capital Medical University, Beijing 100053; \\ ${ }^{2}$ Beijing Institute of Ophthalmology, Beijing Tongren Eye Center, Beijing Tongren Hospital, \\ Capital Medical University, Beijing 100005; ${ }^{3}$ Department of Interventional Radiology, Beijing \\ Xuan Wu Hospital, Capital Medical University, Beijing 100053, P.R. China
}

Received May 28, 2015; Accepted June 23, 2016

DOI: $10.3892 /$ etm.2016.3581

\begin{abstract}
A 70 year old male was diagnosed with carotid stenosis and underwent carotid artery stenting (CAS). Severe postoperative complications arose subsequent to the procedure, including decreased visual acuity and symptoms of a visual field defect, and the patient was eventually diagnosed with retinal artery embolism. CAS reduces carotid plaque; however, the subsequent shedding of carotid plaque may cause retinal artery embolism, resulting in the serious loss of visual function in patients. This complication is of paramount importance and requires great consideration from the neurologist and the patients undergoing CAS. Ophthalmic evaluation is paramount following CAS due to the possibility of embolic occlusion of the retinal artery. It is imperative that patients be informed of the risk of permanent vision loss as a result of CAS.
\end{abstract}

\section{Introduction}

Carotid artery stenosis refers to the stenosis of the lumen owing to carotid atherosclerotic plaque, arteritis, or fibromuscular dysplasia. Its incidence is high, accounting for $10 \%$ of the population $>60$ years old. Carotid artery stenosis is among the leading causes of ischemic stroke and patients usually arrive at neurological clinics with stroke symptoms of hemiplegia and homonymous hemianopia (1). Targeted surgery treatments include carotid endarterectomy (CEA) and CAS. Two types of surgery for the treatment of carotid artery stenosis remain controversial. However, CAS has increasingly become accepted as a mainstay therapy for the treatment of stenosis of the carotid artery bifurcation lesions due to the reduced probability of

Correspondence to: Miss. Xiao-Yan Peng, Beijing Institute of Ophthalmology, Beijing Tongren Eye Center, Beijing Tongren Hospital, Capital Medical University, 17 Houhou Street, Beijing 100005, P.R. China

E-mail: drrpengxy@163.com

Key words: carotid artery stenting, carotid stenosis, retinal artery embolization non-stroke complications and mortality due to CAS trauma (2). However, the resultant shedding carotid plaque may cause retinal artery embolism, resulting in the severe loss of visual function in patients after CAS procedure. The present study describes a case of branch retinal artery occlusion following CAS, reinforcing the necessity for ophthalmic evaluation following the surgery.

\section{Case report}

A 70 year old male presented to the Department of Neurosurgery at Xuanwu Hospital of Capital Medical University (Beijing, China) in April 2014 after having suffered from intermittent dizziness for 6 months. The patient had suffered from hypertension for 20 years and diabetes mellitus for 15 years. An intracarotid angiography revealed severe (99\%) right carotid artery stenosis. Percutaneous transfemoral carotid stenting was performed (Fig. 1). The patient complained of blurred vision and scotoma in the superior visual field of the right eye $8 \mathrm{~h}$ after the procedure. The best corrected visual acuity of the right eye was 0.2 and 0.8 in the left eye. The intraocular pressure was 11 and $12 \mathrm{mmHg}$ in the right and left eye, respectively. Fundus photography revealed retinal whitening and edema in the inferior quadrant of the right eye with partial involvement of the fovea (Fig. 2A). Multiple intra-arterial emboli were discovered and confirmed by fundus fluorescent angiography, fluorescein angiogram indicated delayed filling and emboli and perivascular leaking of the right eye (Fig. 3). Magnetic resonance imaging of the brain revealed multiple new-onset acute cerebral infarctions (Fig. 4). The patient was then administered therapies for the purpose of dilating the blood vessels including regular aspirin and anticoagulants. Visual acuity improved to 0.5 at the final visit 6 months after the procedure, and the fundus photography revealed that the symptoms of retinal whitening and edema had improved. However, visible intravascular emboli and vascular occlusion was still present and are observed as a white line in Fig. 2B.

\section{Discussion}

The retinal artery, as a terminal artery, is the only source of nutrition for the inner retina and there are no anastomoses 



Figure 1 Transfemoral carotid angiography reveals severe occlusion of the internal carotid artery at the (A) proximal branching portion (red arrow) and (B) the improvement of the same site following CAS (blue arrow). CAS, Carotid artery stenosis.
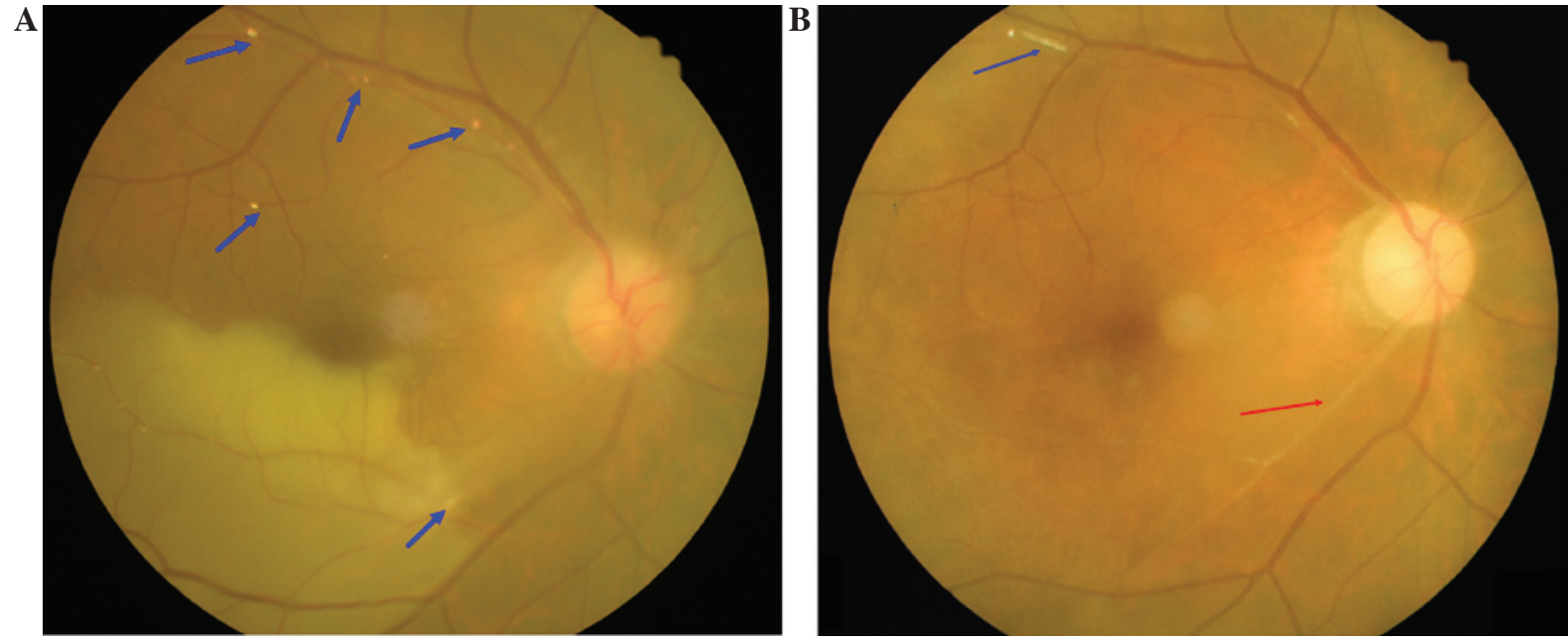

Figure 2. (A) Fundus photography revealed retinal whitening and edema in the inferior quadrant of the right eye and multiple intra-arterial emboli (blue arrows). (B) Six months after the procedure, fundus photography indicated retinal whitening and improved edema. Visible intravascular emboli (blue arrow) and branch vascular occlusion appear as a white line (red arrow).

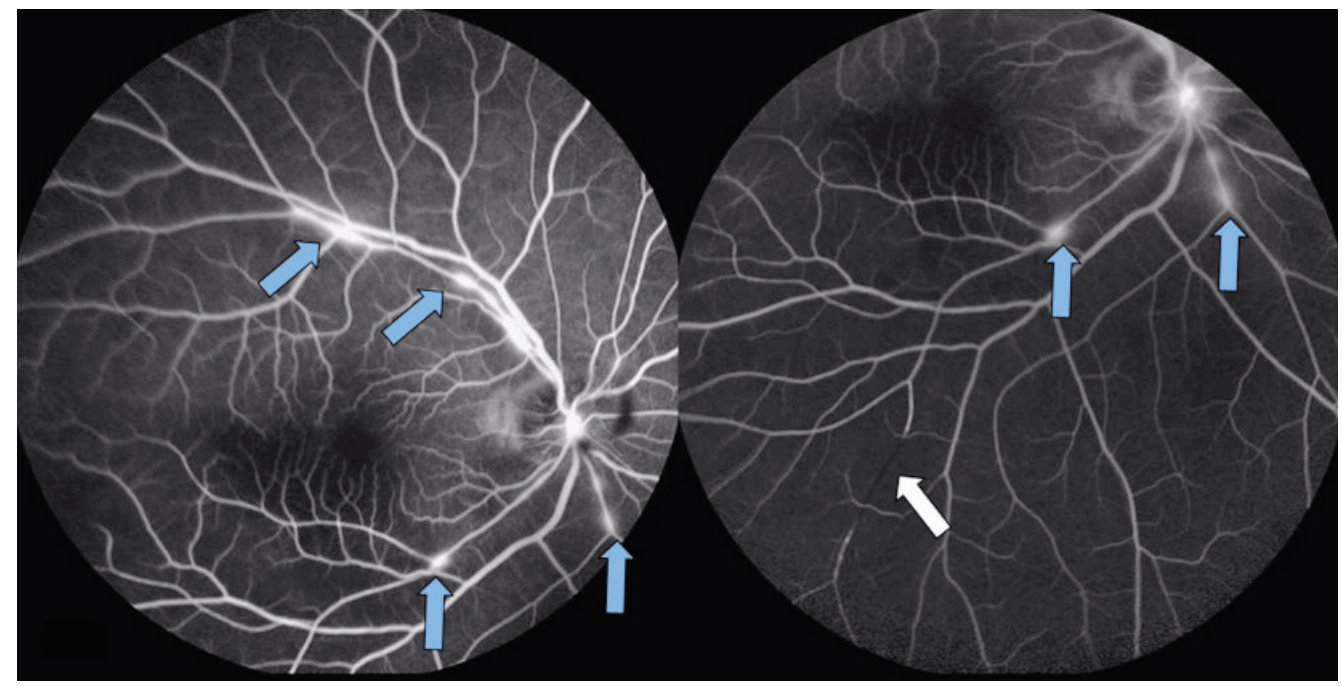

Figure 3. Fluorescein angiogram indicated delayed filling (white arrow), and emboli and perivascular leaking of the right eye (blue arrows). 


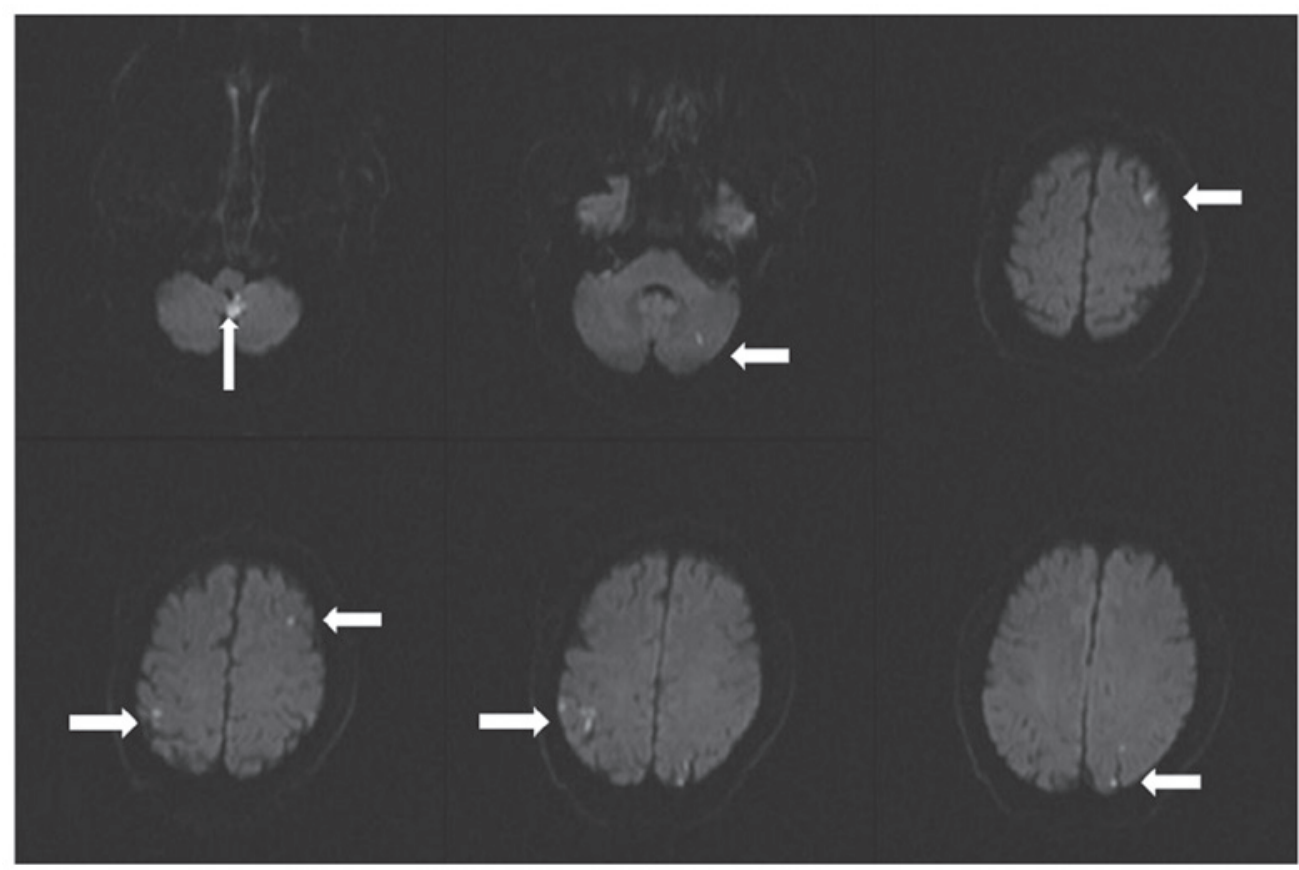

Figure 4. Brain magnetic resonance imaging showed multiple new onset acute cerebral infarction (white arrows).

which exit among its branches. Once occlusion has occurred, the subsequent loss of retinal blood supply may result in a decrease in vision and visual field. Causes of CRAO and BRAO include cardiac disease, retinal artery thrombosis, vascular spasm and vasculitis (3). Embolisms from the carotid bifurcation are considered to be the most common cause of CRAO and BRAO (4). CAS is currently the predominant method employed for the treatment of carotid stenosis; however, the procedure may result in the occlusion of the retinal artery. Retinal artery embolization during CAS is a rare but serious complication that can lead to partial or total blindness (5).

The internal carotid artery (ICA) gives rise to the ophthalmic arteries, therefore ICA stenosis may result in an insufficient blood supply to the inner retina. Thus, retinal artery occlusion may occur as a result of embolisms induced by carotid stenosis, as the flow of blood may flush the debris of atherosclerotic plaque toward the retinal artery. In CAS, debris may be flushed to the retinal artery during the placement of carotid stents and embolic protection devices (EPDs). In addition, the anastomosis of external carotid (ECA) circulation to the internal carotid artery (i.e., from the orbital branch of the middle meningeal artery to ophthalmic artery) may form with carotid stenosis (6), and $8-35 \%$ of preoperative carotid stenosis patients have reverse flow in the ophthalmic artery prior to surgery $(7,8)$.

Transient blindness, amaurosis fugax, has previously been reported in patients with a fully occluded ipsilateral internal carotid, unequivocally indicating the collateral pathway to be a mechanism for retinal embolization (2). The passage of debris through the aforementioned collaterals poses a significant risk as flushing debris through the orbital branch of the middle meningeal artery towards the ECA, in particular, may lead to retinal embolization (9). The absence of EPDs to filter the debris may also lead to retinal embolization following CAS. Additionally, the opening of ECA prior to opening ICA during CAS surgery may result in retinal embolization $(10,11)$.
Embolization resulting in retinal arterial occlusion following CAS surgery, despite the use of EPDs, has been reported in the literature. Vos et al (12) discovered that retinal embolization occurred in 6/118 patients (4\%) undergoing CAS with EPDs, two of whom (1.7\%) were symptomatic. Wilentz et al (2) reported retinal emboli in 5/33 stenting procedure patients (15\%) performed using distal EPDs, two of whom presented with additional small retinal infarcts. The mean rate of embolization per CAS procedure has been reported as 74 particles per stenotic lesion (13). Fortunately, of the embolic material produced following CAS, few are large enough to cause retinal damage; typically, emboli $>20 \mathrm{~mm}$ cause symptomatic retinal artery occlusions in CAS (2).

In the case detailed in the present study, a large number of emboli were observed within the retinal vessels, and a large quantity of emboli were observed which led to intracranial vascular embolism. This conclusion indicated that an increased quantity of embolus is flushed to intracranial vessels in CAS. Investigation into the correlation between retinal artery embolism and intracranial vascular embolism has therefore gained increasing interest. With regard to the aforementioned discoveries, monitoring of patients following CAS is necessary to avoid retinal artery embolism .

The current therapies for the treatment of BRAO and CRAO include the use of acute and chronic measures $(14,15)$. Acute treatment involves modalities which lower the intraocular pressure, including enhanced retinal oxygenation and ocular massage, whilst chronic treatments aim to decrease platelet adhesiveness with therapies such as aspirin, dipyridamole or sulfinpyrazone to prevent the dislodging of further emboli and thrombus formation. However, no therapy has been conclusively demonstrated to be efficacious (16).

In conclusion, ophthalmic evaluation is important following CAS due to the possibility of embolic occlusion of the retinal artery. Patients must be advised of the risk of permanent vision 
loss as a result of CAS, and neurologists performing the procedure are require to be vigilant of this potential postoperative complication of CAS.

\section{References}

1. Faries PL, Chaer RA, Patel S, Lin SC, DeRubertis B and Kent KC: Current management of extracranial carotid artery disease. Vasc Endovascular Surg 40: 165-175, 2006.

2. Wilentz JR, Chati Z, Krafft V and Amor M: Retinal embolization during carotid angioplasty and stenting: Mechanisms and role of cerebral protection systems. Catheter Cardiovasc Interv 56: 320-327, 2002.

3. Kimura K, Hashimoto Y, Ohno H, Uchino M and Ando M Carotid artery disease in patients with retinal artery occlusion. Intern Med 35: 937-940, 1996

4. Hayreh SS, Podhajsky PA and Zimmerman MB: Retinal artery occlusion: Associated systemic and ophthalmic abnormalities. Ophthalmology 116: 1928-1936, 2009.

5. Karjalainen K: Occlusion of the central retinal artery and retinal branch arterioles. A clinical, tonographic and fluorescein angiographic study of 175 patients. Acta Ophthalmol Suppl 109: 1-95, 1971.

6. Cohn EJ Jr, Sandager GP, Benjamin ME, Lilly MP, Hanna DJ and Flinn WR: Assessment of ocular perfusion after carotid endarterectomy with color-flow duplex scanning. J Vasc Surg 29: 665-671, 1999.

7. Costa VP, Kuzniec S, Molnar LJ, Cerri GG, Puech-Leão P and Carvalho CA: The effects of carotid endarterectomy on the retrobulbar circulation of patients with severe occlusive carotid artery disease. An investigation by color Doppler imaging. Ophthalmology 106: 306-310, 1999.
8. Rosenkranz K, Langer R and Felix R: Transcranial Doppler sonography: Collateral pathways in internal carotid artery obstructions. Angiology 42: 819-826, 1991.

9. Sfyroeras GS, Bessias N, Moulakakis KG, Lyra S, Kotsikoris I, Andrikopoulos V and Liapis CD: New cerebral ischemic lesions after carotid endarterectomy. Ann Vasc Surg 27: 883-887, 2013.

10. Madycki G, Staszkiewicz W and Gabrusiewicz A: Carotid plaque texture analysis can predict the incidence of silent brain infarcts among patients undergoing carotid endarterectomy. Eur J Vasc Endovasc Surg 31: 373-380, 2006.

11. Shillito J Jr and Rockett FX: Retinal artery embolism: A complication of carotid endarterectomy. J Neurosurg 20: 718-720, 1963.

12. Vos JA, van Werkum MH, Bistervels JH, Ackerstaff RG, Tromp SC and van den Berg JC: Retinal embolization during carotid angioplasty and stenting: Periprocedural data and follow-up. Cardiovasc Intervent Radiol 33: 714-719, 2010.

13. Jordan WD Jr, Voellinger DC, Doblar DD, Plyushcheva NP, Fisher WS and McDowell HA: Microemboli detected by transcranial Doppler monitoring in patients during carotid angioplasty versus carotid endarterectomy. Cardiovasc Surg 7: 33-38, 1999.

14. Beiran I, Goldenberg I, Adir Y, Tamir A, Shupak A and Miller B: Early hyperbaric oxygen therapy for retinal artery occlusion. Eur J Ophthalmol 11: 345-350, 2001.

15. Rumelt S, Dorenboim Y and Rehany U: Aggressive systematic treatment for central retinal artery occlusion. Am J Ophthalmol 128: 733-738, 1999.

16. Fraser SG and Adams W. Interventions for acute non-arteritic central retinal artery occlusion. Cochrane Database Syst Rev 1: CD001989, 2009. 